\title{
Contemporary neonatal transport: problems and solutions
}

\section{Cornette}

Perinatal health care should be regionalised and this should include a stand alone, reliable, and effective neonatal transport team

W ithin the United Kingdom, ${ }^{12}$ as well as in many other European countries, ${ }^{3}$ it is now accepted that neonatal intensive care should no longer be left to local initiatives, but has to be commissioned on a regional basis. Regionalisation of high risk perinatal health care by establishing and commissioning managed clinical networks aims to maintain equality of access whilst maximising the level of evidence based care and outcome, ${ }^{4}$ working in partnership with agreed patterns of referral and shared protocols. ${ }^{5}$ The aim is a flexible organisation that can respond well to a changing environment. As a result, a stand alone, reliable, and effective neonatal transport team with strong clinical and managerial leadership is considered as a crucial, although not exclusive, component to the success of a network.

Over the past decade, many regions in the United Kingdom have developed excellent neonatal transport teams. Often with very small budgets, teams have successfully put state of the art equipment into daily practice and set local standards for a safe and high quality standard of health care within a changing environment. However, the 2 lst century debate on neonatal transport must touch on issues that are of a broader nature-for example, how must a transport team deal with an ever increasing workload and clinical governance demands? Also, does team configuration need changing within the context of a network, and which new professional roles are needed? In other words, what are today's and tomorrow's solutions for modern or contemporary neonatal transport problems?

\section{REORGANISE THE TRANSPORT LANDSCAPE}

Neonatal transport is a high risk service to work in, because of the type of patients, a changing environment, differing equipment, and a high workload and responsibility. ${ }^{6}$ A preponderance of evidence supports the association of high volume activity with better outcome with respect to a variety of conditions and procedures. It is thus no longer appropriate that small, ad hoc teams undertake occasional, ill prepared, and hazardous transport, often with obsolete equipment and minimal experience. ${ }^{7}$ Transferring critically ill neonates without specialised staff results in greater morbidity and mortality. ${ }^{8}$ For reasons of clinical governance, the only acceptable standard for every managed clinical network is to establish a single, highly trained and professional team that carries out all neonatal transports. As geographical and demographic differences must be taken into account, compromises will have to be made and a measured approach will be needed.

Centralisation of neonatal transport obviously needs a considerable financial injection into existing transport teams. In addition, one must invest in mechanisms that ensure close partnership with local or smaller hospitals, as networking is about collaboration. A priority for modern transport teams is to work in partnership with an outreach, interprofessional educational programme, its aim being twofold. Firstly, outreach education must primarily focus on the early detection of the high risk fetus and maternal referral to a tertiary centre, as antenatal referral remains the goal. Secondly, it must strive towards sharing evidence between local hospitals and develop regional guidelines, preferably starting with targeting high risk areas such as neonatal transport. There can be no network without sharing practice: collaborative working also implies collaborative learning. For instance, the development of regionally accepted guidelines on neonatal stabilisation before transport is a starting point. Its impact can be determined by a systematic audit of referral patterns, standard of care during stabilisation, and neonatal outcome. ${ }^{9}$

\section{CREATE NEW WORKING PATTERNS}

Transport teams are often too heavily dependent on doctors in training, especially out of normal working hours. Hence, expanding the number of staff attached to existing teams may prove to be difficult in view of the imminent reduction in the working hours of junior doctors, as well as the problems of recruiting and retaining nurses. Hence, new working patterns need to be considered, such as neonatal nurse practitioners (NNPs) taking a lead during acute neonatal transports. Although the North American and Canadian systems of nurse led neonatal transport run very efficiently, nurse led transport has been slow to develop in Europe. ${ }^{10}$ Whereas until recently there were few nurse led transport services in the United Kingdom, ${ }^{11}$ the practice is becoming more popular and was recently validated as being practical and safe. ${ }^{12}$ Success of such teams will depend on realistic working patterns, correct patient care responsibilities as determined by individual skill sets, underpinned by good teamwork and sufficient medical advice and collaboration. The worries, however, are their insufficient number and a lack of nationally agreed standards of NNP competency.

Investment in neonatal transport nurses with extended practice has potentially many advantages. Firstly, it is a means of being innovative and facilitative in attracting nurses into neonatal medicine. Neonatal transport is an area in which the NNP can have a pioneering role, as the job creates a stimulating and challenging opportunity for professional growth and development. Secondly, NNPs can have a role in teaching regional guidelines, as implementation and regional ownership of these guidelines requires continuous communication and feedback on performance. Thirdly, NNPs are a valuable resource to the entire health community, and must therefore receive sufficient support for continuous professional development.

Amalgamating neonatal and paediatric teams into a combined neonatal and paediatric service seems the best way to generate a sufficiently high level of activity and to reach excellence. A well documented model of such a generic service is the New South Wales Neonatal and Paediatric Emergency Transport Service, although their demographic situation is very different from other regions in the world. ${ }^{13}$ I would rather see the creation of a generic service as an end point of a stepwise process with a realistic time scale. The main obstacle will be a shortage of doctors, as currently most teams are under pressure to provide rotas that comply with the European Working Time Directive. A reduction in the availability of junior medical staff 
for service provision will undoubtedly contribute to the need for a considerable expansion of the consultant workforce, ${ }^{14}$ whose contribution to neonatal transport should be ever increasing in view of increasing complexity of neonatal care.

\section{ESTABLISH A NATIONAL ADVISORY GROUP FOR NEONATAL TRANSPORT}

In the United Kingdom, neonatal transport services have developed largely as an unplanned response to local service needs. ${ }^{15}$ If neonatal stand alone transport teams concentrate exclusively on the micro level without due regard to the large scale context, team effectiveness and efficiency are likely to be compromised. If we continue to allow each transport team to create its own transport ethos as it goes, there is a clear risk of underestimating the complexity of the network reform ahead. What we therefore urgently need is a sensitive national group that pulls the decentralised clinical teams together to form an effective, corporate whole. Such a national group should draw on the skills and leadership qualities of enthusiastic transport coordinators-that is, doctors and nurses-who regularly meet, discuss locally discovered solutions, and develop national guidance. Success will depend on constructive dialogue, improved understanding, mutual respect, and the maturity to live with differing points of view.

The scope of activity for such an advisory group is wide ranging. Firstly, the group must ensure that standards for safe neonatal transport are set at a national level. For instance, clinical standards for transport that might be agreed at a national level and used locally as benchmarks include response time, successful airway management, patient satisfaction, compliance with protocols, and efficiency of continuing educational processes for team members. ${ }^{16}$ Other standards include: (a) clarification on competencies required for transport-that is, clinical, procedural, or equipment related; $(b)$ guidance on appropriate team configuration (nurse, NNP, doctor, paramedics) according to the type of transfer (acute or non-acute); (c) an inventory on currently operating transport teams/ strategies and guidance on team structure according to demographics-for example, not every network needs air transport facilities; $(d)$ the development of clinical guidelines; $(e)$ clear referral criteria or policies-for example, which of all antenatally diagnosed congenital abnormalities need to deliver in a tertiary care centre (that is, involve obstetricians) and how many emergency bed bureaus (that is, facilities that help to locate cots and transport infants) do we need in a country? As such, it is hoped that regionalisation of neonatal care will stimulate the development of clear care pathways.

Secondly, the advisory group must ensure that clinical standards are delivered and monitored. The tools are audit, clinical risk management, and research. It requires everyone to constantly question what they are doing and why. If transport teams are to have an impact on neonatal outcome, they must constantly query whether or not each transfer was essential and appropriate. The importance of research in neonatal transport is currently underestimated, and the development of centralised transport teams across a network of collaborating neonatal units provides us with a unique opportunity to organise multicentre scaled projects, investigating important questions on clinical, organisational, economic, or teaching aspects in neonatal transport. For instance, there is a need to evaluate the cost/benefit of existing neonatal intensive care treatments-for example, nitric oxide, head cooling, high frequency oscillation, and mobile extracorporeal membrane oxygenationbefore automatically extending them to the transport environment. ${ }^{16}$ Grouping of small scale projects can contribute to more generalised learning and inform scientific knowledge about quality improvement in neonatal transport.

Thirdly, there is a clear task for such a group to look into legality aspects of regional transport, regulations, accountability, etc, in order to minimise the risk of litigation and to provide jurisprudence for all teams at various time points during transport.

Fourthly, there is a need to better understand the economics of neonatal transport, as limited understanding of team costs and revenue streams may threaten the survival of transport services. ${ }^{16}$

Finally, it is impossible to reform a healthcare system with single initiatives. Multifaceted strategies are needed and can be coordinated by such an advisory group. The group should collaborate closely with other national neonatal groups in order to jointly address and clarify neonatal network issues such as the impact of the new classification of units with respect to neonatal transport $^{17}$ (for example, expected increase in rapid return transfers when intensive care is no longer required), patient flows, and accessibility of networks, neonatal workforce planning (that is, an exceptionally complex task, examining education, training, skill mix, recruitment and retention, and career structure), and resources in relation to network performance.

It thus becomes clear that, for all the reasons described, a national advisory group on neonatal transport will help networks to move away from historical or political positioning but come together and collaborate. I strongly believe that a national debate can result in a more coherent strategy and hence increased resources. However, elegant and imaginative reforms at a higher level are of no value if they do not translate into a public perception that things are better. The aim must therefore be to produce detailed project plans, implement incremental refinementsnot a complete redesign-and, above all, make things happen. National collaboration will also provide us with the opportunity to better understand the concepts of quality, cost containment, and the relation between them. Only then can we reach a consensus about the level of expenditure a nation can afford or wants to spend on neonatal transport to improve outcome of care.

\section{EXPAND MODERNISATION STRATEGIES}

The sector of neonatal transport has not seen an explosion of scientific information over the past decade. However, evidence based medicine does not exclusively require randomised controlled trials. A good evidence base also comprises the explicit synthesis of the best available external evidence and clinical expertise in the provision of care for individual patients. We cannot rely on assumptions, as such will never lead to progress. Transport teams need clear agreement on collecting a standard minimum dataset for transport-for example, type of transfers, refused transfers, demographic data, etctogether with a transparent data analysis. A uniform approach to data collection will facilitate quality improvement by highlighting regional differences in outcome, shortfalls in standards, comparisons with other services and time trends - that is, the basis of benchmarking. In addition, continuous critical evaluation of the data will inform future service modelling.

Transport teams need to develop audit and research through partnership with universities and other networks. Constructive engagement will be crucial, as will be the creation of a platform for dialogue and consensus.

Transport teams need to be open to new technology that facilitates collaboration. Hence, investment in computerised infrastructure is essential. For instance, telemedicine is a valuable resource that allows patient triage through video conferencing, or consultation and 
advice when healthcare providers are not close by. Advanced methods of communication are likely to allow a more appropriate allocation of team resources.

Finally, we need more media coverage on the excellent work already done by many teams, in order to deserve the same public support as the latest biomedical breakthroughs.

\section{CONCLUSION}

The current climate of change in health care is driven by political priorities, cost control efforts, rising public expectations, and considerable progress in science, computer, and communication technology. It therefore becomes clear that future resources will preferentially go into new models of working patterns that are evidence based. Short term solutions will always create long term problems. Neonatal transport teams thus cannot be happy with status quo or mediocrity, but need to make a difference and achieve excellence. As the provision of neonatal intensive care is changing across Europe, neonatal transport teams must not discard this opportunity to be creative and innovative, because the changes will be worth while.
Arch Dis Child Fetal Neonatal Ed 2004;89:F212-F214

doi: 10.1136/adc.2003.046201

Correspondence to: Dr Cornette, Yorkshire Region Neonatal Transport Service, Peter Congdon Neonatal Unit, Clarendon Wing C Floor, Leeds General Infirmary, Great George Street, Leeds LS2 9NS, UK

luc.cornette@lth.nhs.uk

\section{REFERENCES}

1 Department of Health. Getting the right start: National Service Framework for Children, Young People and Maternity Services. Part 1: the NSF Emerging Findings consultation document. London: Department of Health, 2003. www.doh.gov.uk/NSF/children/ keydocuments.htm.

2 Department of Health. Report of $\mathrm{DoH}$ expert working group on Neonatal Intensive Care Services. London: Department of Health, 2003. www.doh.gov.uk/NSF/children/ keydocuments. htm

3 Agostino R, Antsaklis, Bréart G, et al. (EUROPET expert group). New decision rules: regionalization in perinatal care and indications for perinatal transfer. Prenat Neonatal Med 1999:4:104-7.

4 Department of Health. The report of the public inquiry into children's heart surgery at the Bristol Royal Infirmary 1984-1995 learning from Bristol. Published by the Bristol Royal Infirmary Inquiry, July 2001. www. bristol-inquiry.org.uk/final_report.

5 Cesdi, Project 27/28. An enquiry into quality of care and its effect on the survival of babies born at 27-28 weeks.

6 Barry PW, Ralston C. Adverse events occurring during interhospital transfer of the critically ill. Arch Dis Child 1994;71:8-11.
7 Parmanum J, Field D, Rennie J, et al National census of availability of neonatal intensive care. BMJ 2000;321:727-9.

8 Agostino R, Fenton AC, Kollée LAA, et al. Organization of neonatal transport in Europe. Prenat Neonatal Med 1999;4:20-34.

9 Shenai JP. Neonatal transport. Outreach educational program. Pediatr Clin N Am 1993:40:275-85.

10 King BR, Foster RL, Woodward GA, et al. Procedures performed by pediatric transport nurses: how "advanced" is the practice? Pediatr Emerg Care 2001;17:410-13.

11 Watts C. The role of the ANNP: nurse-led neonatal transports. J Neonatal Nurs 2001;7:196-200.

12 Leslie A, Stephenson T. Neonatal transfers by advanced neonatal nurse practitioners and paediatric registrars. Arch Dis Child Fetal Neonatal Ed 2003;88:F509-12.

13 Rashid A, Bhuta T, Berry A. A regionalised transport service, the way ahead? Arch Dis Child 1999:80:488-92.

14 Scott-Coombes D, Chan YC. European working time directive for doctors in training. BMJ 2002;324:736.

15 Papiernik E, Zeitlin J, Milligan DWA, et al. Variations in the organization of obstetric and neonatal intensive care in Europe. Prenat Neonatal Med 1999;4:73-87.

16 Woodward GA, Insoft RM, Pearson-Shaver AL, et al. The state of pediatric interfacility transport: consensus of the second national pediatric and neonatal interfacility transport medicine leadership conference. Pediatr Emerg Care 2002; 18:38-43.

17 British Association of Perinatal Medicine. Standards for hospitals providing neonatal intensive care and high dependency care and categories of babies requiring neonatal care. 2nd ed. London: British Association of Perinatal Medicine, 2001 (www.bapm-London.org) 